\title{
Antimicrobial susceptibility patterns, emm type distribution and genetic diversity of Streptococcus pyogenes recovered in Brazil
}

\author{
Glauber P Arêas, Rôde BB Schuab, Felipe PG Neves, Rosana R Barros/+ \\ Departamento de Microbiologia e Parasitologia, Instituto Biomédico, Universidade Federal Fluminense, Niterói, RJ, Brasil
}

\begin{abstract}
Streptococcus pyogenes is responsible for a variety of infectious diseases and immunological complications. In this study, 91 isolates of S. pyogenes recovered from oropharynx secretions were submitted to antimicrobial susceptibility testing, emm typing and pulsed-field gel electrophoresis (PFGE) analysis. All isolates were susceptible to ceftriaxone, levofloxacin, penicillin $G$ and vancomycin. Resistance to erythromycin and clindamycin was $15.4 \%$, which is higher than previous reports from this area, while $20.9 \%$ of the isolates were not susceptible to tetracycline. The macrolide resistance phenotypes were $c M L S_{B}(10)$ and $i M L S_{B}(4)$ The $\mathrm{ermB}$ gene was predominant, followed by the ermA gene. Thirty-two emm types and subtypes were found, but five (emm1, emm4, emm12, emm22, emm81) were detected in $48 \%$ of the isolates. Three new emm subtypes were identified (emm1.74, emm58.14, emm76.7). There was a strong association between $\mathrm{emm}$ type and PFGE clustering. A variety of PFGE profiles as well as emm types were found among tetracycline and erythromycin-resistant isolates, demonstrating that antimicrobial resistant strains do not result from the expansion of one or a few clones. This study provides epidemiological data that contribute to the development of suitable strategies for the prevention and treatment of such infections in a poorly studied area.
\end{abstract}

Key words: Streptococcus pyogenes - antimicrobial resistance - epidemiological typing - genetic diversity

Streptococcus pyogenes [Group A Streptococcus (GAS)] is one of the most clinically relevant species of Streptococcus. It has been associated with human infections ranging from mild sore throat and impetigo to invasive, life-threatening diseases, such as necrotising fasciitis and toxic shock syndrome. Moreover, immunological complications such as acute rheumatic fever, rheumatic heart disease and post-streptococcal acute glomerulonephritis are significant streptococcal disease burdens, especially in the developing world (Steer et al. 2009, Dale et al. 2013).

Several efforts have been made over the past decades to understand the epidemiology of GAS infections. Contributing to these efforts, molecular techniques such pulsed-field gel electrophoresis (PFGE) and multilocus sequencing typing have been extensively applied in the evaluation of the genetic relationships among isolates (Dicuonzo et al. 2001, Ardanuy et al. 2010). Another approach is emm typing, a sequence-based typing of the M protein (Beall et al. 1996). M protein is a cell surface protein that inhibits phagocytosis and is considered the most important epidemiological marker of GAS infections. This approach has the potential to type GAS isolates from different areas of the world. However, most available data are from high-income countries, which has lead to several limitations, such as the lack of knowledge

doi: $10.1590 / 0074-0276140231$

Financial support: PROPPI/UFF, FAPERJ

+ Corresponding author: miprosana@vm.uff.br

Received 27 June 2014

Accepted 25 September 2014 of emm type prevalence in developing countries where severe streptococcal infections and immunological complications occur frequently (Steer et al. 2009).

The development of an efficient vaccine for GAS infections is another important issue. Several candidates have been evaluated, including the variable $\mathrm{N}$-terminal and the conserved $\mathrm{C}$-terminal epitopes of $\mathrm{M}$ protein (McNeil et al. 2005, Dale et al. 2011, Postol et al. 2013). Knowledge of the global distribution of emm types is required to formulate an $\mathrm{N}$-terminal $\mathrm{M}$ protein-based vaccine that covers the prevalent $M$ types in different geographical areas (Steer et al. 2009).

Treatment of GAS infections is based on beta-lactams because this species remains susceptible to penicillin G; macrolides, lincosamides and fluoroquinolones are recommended for allergic individuals. However, GAS resistance to these later antimicrobials has been described worldwide (Smeesters et al. 2009, Friães et al. 2012). While fluoroquinolone resistance in GAS is due to point mutations in the gyr and par genes, macrolide resistance is due to active efflux (M phenotype) or modification of the target site (iMLS $\mathrm{B}_{\mathrm{B}}$ and $\mathrm{CMLS}_{\mathrm{B}}$ phenotypes) and is mediated by the genetic determinants mefA/E, ermA and ermB (D`Oliveira et al. 2003, Montes et al. 2010). It is worth noting that in some countries where macrolide resistance rates used to be high, such as Spain and China, unexpected reductions in the resistance rates have been recently observed (Huang et al. 2014, Montes et al. 2014).

Antimicrobial resistance rates and emm type distribution in a given population are essential to the development of suitable strategies for the prevention and treatment of GAS infections. The aim of this study was to investigate the antimicrobial susceptibility and emm type and to evaluate the genetic diversity of circulating GAS isolates in the metropolitan area of Rio de Janeiro, Brazil. 


\section{SUBJECTS, MATERIALS AND METHODS}

Isolates - Ninety-one GAS isolates recovered from oropharynx secretions were included in this study. Clinical specimens were processed during routine diagnoses by one clinical laboratory in Rio de Janeiro from January 2008July 2012. The subjects' ages varied from two-56 years, but $46 \%$ of the isolates were recovered from children two11 years of age and $51.6 \%$ were from women. In our laboratory, these isolates were cultured on blood agar plates (Difco Laboratories, USA) and submitted to conventional tests (PYR test, bacitracin susceptibility and streptococcal serogrouping) to confirm species identification.

Antimicrobial susceptibility testing - All isolates were submitted to susceptibility tests to ceftriaxone (30 $\mu \mathrm{g})$, clindamycin $(2 \mu \mathrm{g})$, erythromycin $(15 \mu \mathrm{g})$, levofloxacin $(5 \mu \mathrm{g})$, penicillin $(10 \mathrm{U})$, tetracycline $(30 \mu \mathrm{g})$ and vancomycin $(30 \mu \mathrm{g})(\mathrm{CECON}$, Brazil) using the disk diffusion method on Mueller-Hinton blood agar (Difco) according to CLSI guidelines (2013). Macrolide resistance phenotypes were determined by the double disk test using erythromycin $(15 \mu \mathrm{g})$ and clindamycin $(2 \mu \mathrm{g})$ disks placed $12 \mathrm{~mm}$ apart (CLSI 2013). The erythromycin minimum inhibitory concentration (MIC) was determined in all resistant and intermediate isolates by the agar dilution method (CLSI 2009).

Investigation of erythromycin resistance-associated genes - DNA preparation was performed as previously described (Dmitriev et al. 2002) with modifications. Briefly, suspensions with turbidity adjusted to McFarland Standard 3.0 were prepared in $300 \mu \mathrm{L}$ of $10 \mathrm{mM}$ Tris-EDTA buffer and boiled for $5 \mathrm{~min}$. The presence of ermA, ermB and $m e f A / E$ genes was investigated in erythromycin-resistant isolates using specific polymerase chain reaction (PCR) protocols (Sutcliffe et al. 1996, Perez-Trallero et al. 2007). Cycling was carried in a GeneAmp 9700 Thermocycler (Applied Biosystems, USA). PCR products were resolved on $1 \%$ agarose gels.

Determination of emm types - emm types were determined by a sequence-based protocol (cdc.gov/ncidod/ biotech/strep/protocol_emm-type.htm) using BigDye Terminator Cycle Sequencing Kit (Applied Biosystems). Sequencing was performed using a 3130 Genetic Analyzer (Applied Biosystems). Sequences were edited using Bioedit software v.7.0 and compared with reference sequences using the BLAST algorithm (blast.ncbi.nlm.nih.gov/Blast. cgi). Sequences that did not match with $100 \%$ similarity to any sequence deposited in GenBank were submitted to the Centers for Disease Control and Disease (CDC) emm sequence database (cdc.gov/ncidod/biotech/strep/strepblast.htm) for assignment to new emm subtypes.

Analysis of DNA restriction patterns by PFGE - All 44 isolates belonging to the five prevalent emm types (emm1, emm4, emm12, emm22 and emm81) were analysed by PFGE after the DNA was digested with SmaI according to a previous protocol (Teixeira et al. 1997) with modifications as described below. Briefly, bacteria were grown on blood agar plates. A $300-\mu \mathrm{L}$ aliquot of bacterial suspension in PIV buffer was mixed with an equal volume of low melting point agarose (Promega, USA) and distributed into plug moulds. Plugs were incubated in $2 \mathrm{~mL}$ of lysis solution containing $5 \mathrm{mg} / \mathrm{mL}$ lysozyme. SmaI digestion was performed according to the manufacturer's recommendations (Invitrogen, USA). DNA fragments were separated by the CHEF-DRIII system (Bio-Rad Laboratories, USA). The dice coefficient was calculated by visual analysis and dendrograms based on Unweighted Pair Group Method with Arithmetic Mean were constructed using genomes.urv.cat/UPGMA.

Statistical analyses - The discriminatory power of emm typing, regarding the overall population as well as erythromycin and tetracycline resistance, was measured using Simpson's index of diversity (SID) by calculating the $95 \%$ confidence intervals (CI) (Hunter \& Gaston 1988, Grundmann et al. 2001). All calculations were performed using the Comparing Partitions Tool available from comparingpartitions.info.

\section{RESULTS}

Conventional tests identified all isolates as S. pyogenes. By the disk diffusion method, all isolates were susceptible to ceftriaxone, levofloxacin, penicillin $\mathrm{G}$ and vancomycin. Tetracycline-resistant and intermediate isolates comprised $18.7 \%$ and $2.2 \%$ of isolates, respectively. Clindamycin resistance was observed in $15.4 \%$ of isolates, while erythromycin-resistant and intermediate isolates comprised $14.3 \%$ and $1.1 \%$ of isolates, respectively. The erythromycin MIC varied from 8-256 $\mu \mathrm{g} /$ $\mathrm{mL}$ and therefore, the resistance rate was $15.4 \%$. Ten and four isolates showed $\mathrm{cMLS}_{\mathrm{B}}$ and $\mathrm{iMLS}_{\mathrm{B}}$ resistance phenotypes, respectively. The genetic determinant ermB was predominant and was detected in $78.6 \%$ of the erythromycin-resistant isolates, alone or in association with ermA (64.3\%). Neither the M phenotype nor the mefA/E gene was observed in this study. Erythromycin MIC values, the distribution of macrolide resistance phenotypes and genotypes and emm types of erythromycin-resistant isolates are shown in Table.

Thirty-two emm types or subtypes were identified among 86 of the 91 GAS isolates. Five isolates were nontypeable, even after three attempts. The most frequent

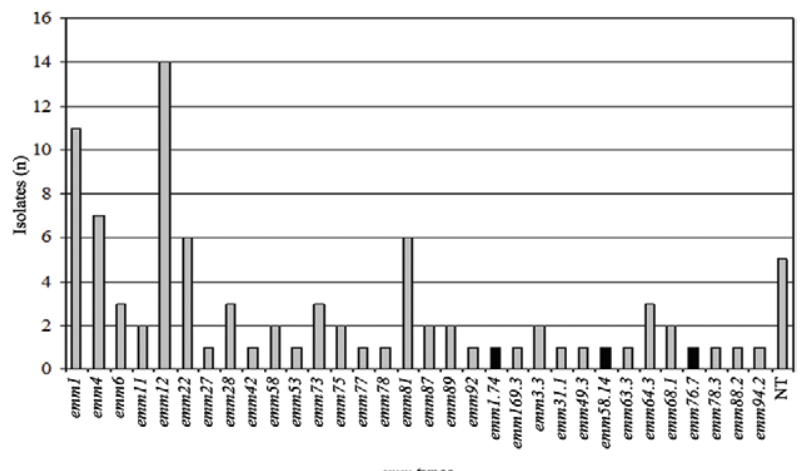

emm types

Fig. 1: distribution of emm types and subtypes among isolates of Streptococcus pyogenes recovered from oropharynx secretion. NT: non-typeable; $\mathbf{n}$ : new subtypes. 
TABLE

Phenotypic and genotypic characteristics of macrolide resistant isolates

\begin{tabular}{cccccccc}
\hline & & & & \multicolumn{3}{c}{ Macrolide resistance genes } \\
\cline { 6 - 8 } $\begin{array}{c}\text { Isolate } \\
\text { number }\end{array}$ & $\begin{array}{c}\text { Year of } \\
\text { recovery }\end{array}$ & $\begin{array}{c}e m m \\
\text { type }\end{array}$ & $\begin{array}{c}\text { MIC } \\
(\mu \mathrm{g} / \mathrm{mL})\end{array}$ & Phenotype & ermA & ermB & mefA/E \\
\hline 186 & 2008 & 68.1 & $>256$ & $\operatorname{cMLS}_{\mathrm{B}}$ & - & + & - \\
274 & 2008 & 58 & $>256$ & $\operatorname{iMLS}_{\mathrm{B}}$ & + & - & - \\
302 & 2008 & 58 & $>256$ & $\operatorname{iMLS}_{\mathrm{B}}$ & + & - & - \\
402 & 2009 & 1 & $>256$ & $\operatorname{cMLS}_{\mathrm{B}}$ & - & + & - \\
418 & 2009 & 58.14 & 8 & $\operatorname{iMLS}_{\mathrm{B}}$ & + & - & - \\
$425^{a}$ & 2009 & 3.3 & 16 & $\operatorname{cMLS}_{\mathrm{B}}$ & - & + & - \\
536 & 2010 & 22 & $>256$ & $\operatorname{cMLS}_{\mathrm{B}}$ & - & + & - \\
$637 \mathrm{~A}$ & 2011 & 1 & $>256$ & $\operatorname{cMLS}_{\mathrm{B}}$ & + & + & - \\
638 & 2011 & 76.7 & $>256$ & $\operatorname{cMLS}_{\mathrm{B}}$ & + & + & - \\
710 & 2011 & 28 & $>256$ & $\operatorname{cMLS}_{\mathrm{B}}$ & - & + & - \\
749 & 2011 & 73 & $>256$ & $\operatorname{cMLS}_{\mathrm{B}}$ & + & + & - \\
750 & 2011 & 6 & $>256$ & $\operatorname{cMLS}_{\mathrm{B}}$ & + & + & - \\
780 & 2012 & 11 & $>256$ & $\operatorname{cMLS}_{\mathrm{B}}$ & + & + & - \\
798 & 2012 & 11 & $>256$ & $\operatorname{iMLS}_{\mathrm{B}}$ & + & + & - \\
\hline
\end{tabular}

$a$ : this isolate was intermediate by disk diffusion; CMLS $_{\mathrm{B}}$ : constitutive MLS $_{\mathrm{B}}$ phenotype; iMLS $\mathrm{B}_{\mathrm{B}}$ inducible MLS $_{\mathrm{B}}$ phenotype; MIC: minimal inhibitory concentration (susceptible $<0.25 \mu \mathrm{g} / \mathrm{mL}$, intermediate $0.5 \mu \mathrm{g} / \mathrm{mL}$, resistant $>1 \mu \mathrm{g} / \mathrm{mL}$ ); + : presence of the gene; -: absence of the gene.

types were emm1, emm4, emm12, emm22 and emm81, accounting for $48 \%$ of all isolates. Three new sequences were designated by the CDC Streptococcus Laboratory as new emm subtypes (emm1.74, emm58.14 and emm76.7) and deposited in the emm sequence database (cdc.gov/pub/infectious_diseases/biotech/tsemm) and in GenBank (accession KM364527-KM364529). These new subtypes differed from their parental types in 1-2\% of the nucleotide sequence. The emm typing revealed a high level of diversity among the overall population (SID $=0.941 ; 95 \% \mathrm{CI}, 0.917-0.965)$. The frequency of each emm type is shown in Fig. 1.

Fourteen different emm types or subtypes were found among 19 tetracycline non-susceptible isolates, while 11 distinct emm types or subtypes were found among 14 erythromycin and clindamycin-resistant isolates. Two new emm subtype isolates were resistant to these antimicrobials. The SID values calculated for erythromycinresistant $(0.967 ; 95 \%$ CI, 0.929-1.000) and tetracycline non-susceptible $(0.959 ; 95 \% \mathrm{CI}, 0.915-1.000)$ isolates were higher than those calculated for erythromycin (0.929; 95\% CI, 0.898-0.960) and tetracycline (0.898; $95 \%$ CI, 0.855-0.940) susceptible isolates.

PFGE analysis of 44 isolates belonging to the five prevalent emm types generated 20 restriction profiles, which are shown in Fig. 2. One single PFGE profile was shared by all emm 12 isolates. Three profiles, whose similarities varied from $50-85 \%$, were observed among emml isolates. The most frequent profile was shared by eight isolates, including one resistant to erythromycin. Regarding emm4, four profiles were observed among seven isolates, ranging from $85-96 \%$ similarity. Among emm22 and emm81 isolates (6 isolates each), unique PFGE profiles were observed for each isolate, varying from $70-95 \%$ similarity.

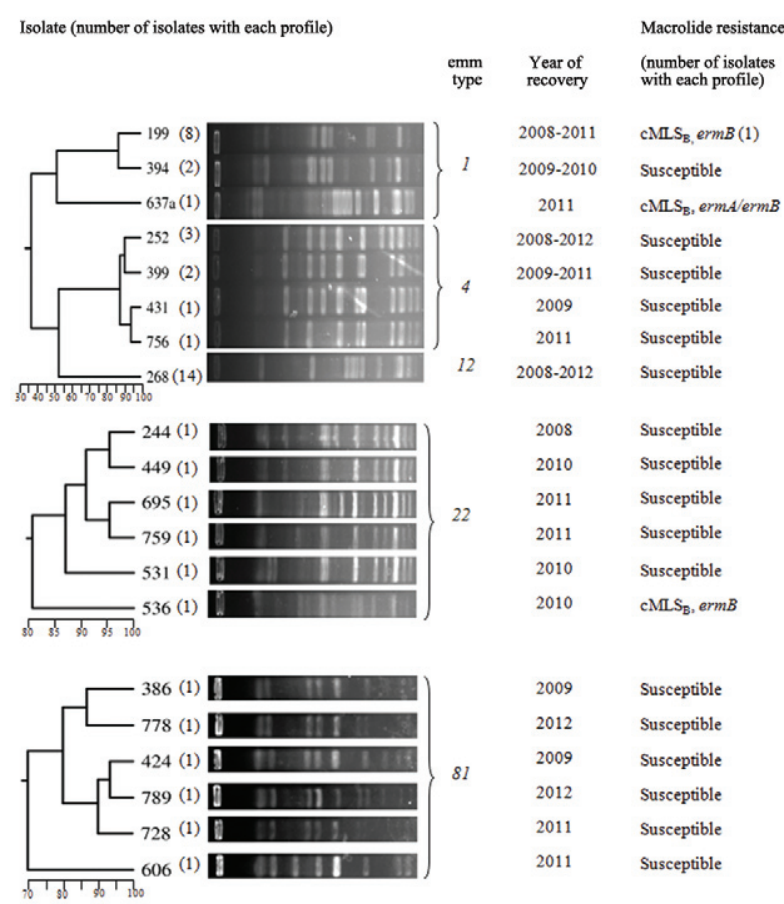

Fig. 2: pulsed-field gel electrophoresis profiles, dendrograms, year of isolation and macrolide resistance features of prevalent $\mathrm{emm}$ types isolates. 


\section{DISCUSSION}

In this study, 91 GAS isolates recovered from oropharynx secretions of residents of Rio de Janeiro were submitted to susceptibility testing and typing methodologies. Despite the lack of data regarding the clinical conditions of the individuals, these results are relevant because both infected and colonised individuals can transmit this species to susceptible subjects.

Isolates were fully susceptible to beta-lactams and glycopeptides, as observed over decades of antibiotic usage. They were also susceptible to levofloxacin, despite previously detected resistance to this antimicrobial (Smeesters et al. 2009, Montes et al. 2010).

The tetracycline resistance rate was lower than that observed in a study conducted in Brazil one decade ago, in which the authors reported $43.1 \%$ resistance (D`Oliveira et al. 2003). Instead, it was very similar to recent data from southern Brazil and Portugal (Torres et al. 2011, Friães et al. 2012). However, 73\% resistance to this antimicrobial was reported in a recent study from India (Mathur et al. 2014). These findings may reflect that, while a consistent trend of decreasing tetracycline resistance has been recently observed in Brazil, circulating GAS isolates from other regions are highly resistant to this antimicrobial.

Regarding erythromycin and clindamycin resistance, the rates found here far exceeded those previously reported in the same geographical area (D`Oliveira et al. 2003, Torres et al. 2011), but they are very similar to those described in some recent studies conducted in Europe (Friães et al. 2012, Syrogiannopoulos et al. 2013). While a trend of decreasing erythromycin resistance has been recently observed in Spain and Taiwan (Huang et al. 2014, Montes et al. 2014), the resistance rates in our study fluctuated over the years. Inducible and constitutive $\mathrm{MLS}_{\mathrm{B}}$ phenotypes, which are associated with ermA and/ or erm $B$ genes, were observed among isolates belonging to a variety of emm types, reflecting the polyclonal origin of such isolates. It is worth noting that the M phenotype and the mefA/E genotype, associated with emm12, were common in Brazil before the year 2000 (Torres et al. 2011), but they have not been detected since that time, either by those authors or in this study. In contrast, MLS $_{B}$ emerged after the 2000s and is linked to a variety of emm types, such as emm11, emm22, emm28 and emm73 (Torres et al. 2011). In contrast to this local replacement of the $\mathrm{M}$ phenotype with the $\mathrm{cMLS}_{\mathrm{B}}$ phenotype, the $\mathrm{M}$ phenotype has been prevalent over 12 years of survey (1998-2010) in Taiwan (Huang et al. 2014). However, the authors observed the replacement of emm12 with emm 22 as the prevalent type associated with macrolide resistance, as well as a decreasing rate in macrolide resistance from $53.1 \%$ before 2000 to $10.7 \%$ from $2006-2010$. These variations demonstrate how dynamic the bacterial population is worldwide and also highlight the need for changing therapeutic recommendations in geographical areas where $\mathrm{MLS}_{\mathrm{B}}$ phenotypes predominate.

A wide variety of emm types, including new alleles, were observed in our study, as demonstrated by the SID calculation. The diversity of emm types and the detection of new alleles from GAS isolates recovered from oropharynx secretions have been described in previous studies from Brazil (Teixeira et al. 2001, Tartof et al. 2010) and throughout the world (Shea et al. 2011). These findings reflect the genetic heterogeneity of such isolates and contribute to the expansion of the emm type database. The most frequent emm types found here are among the prevalent types in high-income countries, where the majority of studies have been performed (Steer et al. 2009, Shea et al. 2011). However, they differ significantly from those types observed in recent studies from India and the Pacific Region (Baroux et al. 2014, Mathur et al. 2014). Due to the limited amount of data from Latin America, our results contribute to a better understanding of emm type distribution in a poorly studied area. This issue is particularly important considering that $\mathrm{M}$ protein-based vaccines are under development (Dale et al. 2013). When comparing the two most promising 26 and 30 -valent $M$ vaccines, $49 \%$ and $64 \%$ of our isolates, respectively, have emm types included in these formulations. The potentially low impact of these vaccines can be explained by the fact that the $M$ protein fragments for the 26-valent $M$ vaccine were selected based on $\mathrm{emm}$ type distribution in North America (Steer et al. 2009).

There was strong agreement between emm type and PFGE clustering, as previously observed (Torres et al. 2011). No identical PFGE profile was observed among isolates belonging to distinct emm types, which could be related to the horizontal transfer of emm genes (Whatmore et al. 1994). Regarding erythromycin-resistant isolates, a great diversity of PFGE profiles and emm types were observed, illustrating that this characteristic is not due to the expansion of a specific clone. Moreover, both erythromycin-susceptible and erythromycin-resistant emml isolates shared a single PFGE profile. This finding suggests that the emm type, not erythromycin resistance, is more likely to be a determinant of clonality.

In conclusion, susceptibility testing and epidemiological typing techniques revealed an incidence of macrolide resistance not yet observed in this area and a great diversity of emm types, including new alleles, among GAS isolates circulating in Brazil. These data contribute to the improvement of prevention and treatment strategies of GAS infections.

\section{ACKNOWLEDGEMENTS}

To the Fleury Group, for strains donation, and to André V Barbosa, from the DNA Sequencing Platform, UFF.

\section{REFERENCES}

Ardanuy C, Domenech A, Rolo D, Calatayud L, Tubau F, Ayats J, Martin R, Liñares J 2010. Molecular characterization of macrolide- and multidrug-resistant Streptococcus pyogenes isolated from adult patients in Barcelona, Spain (1993-2008). J Antimicrob Chemother 65: 634-643.

Baroux N, D‘Ortenzio E, Amédéo N, Baker C, Alsuwayyid BA, Dupont-Rouzeyrol M, O‘Connor O, Steer A, Smeesters PR 2014. The emm-cluster typing system for group A Streptococcus identifies epidemiologic similarities across the Pacific Region. Clin Infect Dis 59: e84-e92.

Beall B, Facklam R, Thompson T 1996. Sequencing emm-specific PCR products for routine and accurate typing of group A streptococci. J Clin Microbiol 34: 953-958. 
CLSI - Clinical Laboratory Standard Institute 2009. Methods for dilution antimicrobial susceptibility tests for bacteria that grow aerobically, M07-A8, 8th ed., CLSI, Wayne, 65 pp.

CLSI - Clinical Laboratory Standard Institute 2013. Performance standards for antimicrobial susceptibility testing, M100-S23, CLSI, Wayne, $199 \mathrm{pp}$.

D`Oliveira REC, Barros RR, Mendonça CRV, Teixeira LM, Castro ACD 2003. Antimicrobial susceptibility and survey of macrolide resistance mechanisms among Streptococcus pyogenes isolated in Rio de Janeiro, Brazil. Microb Drug Resist 9: 87-91.

Dale JB, Fischetti VA, Carapetis JR, Steer AC, Sow S, Kumar R, Mayosi BM, Rubin FA, Mulholland K, Hombach JM, Schödel F, Henao-Restrepo AM 2013. Group A streptococcal vaccines: paving a path for accelerated development. Vaccine 31 (Suppl. 2): B216-B222.

Dale JB, Penfound TA, Chiang EY, Walton WJ 2011. New 30-valent M protein-based vaccine evokes cross-opsonic antibodies against nonvaccine serotypes of group A streptococci. Vaccine 29: 8175-8178.

Dicuonzo G, Gherardi G, Lorino G, Angeletti S, de Cesaris M, Fiscarelli E, Bessen D, Beall B 2001. Group A streptococcal genotypes from pediatric throat isolates in Rome, Italy. J Clin Microbiol 39: 1687-1690.

Dmitriev A, Shakleina E, Tkáciková L, Mikula I, Totolian A 2002. Genetic heterogeneity of the pathogenic potentials of human and bovine group B streptococci. Folia Microbiol (Praha) 47: 291-295.

Friães A, Pinto FR, Silva-Costa C, Ramirez M, Melo-Cristino J, The Portuguese Group for the Study of Streptococcal Infections 2012. Group A streptococci clones associated with invasive infections and pharyngitis in Portugal present differences in emm types, superantigen gene content and antimicrobial resistance. $B M C$ Microbiol 12: 280.

Grundmann H, Hori S, Tanner G 2001. Determining confidence intervals when measuring genetic diversity and the discriminatory abilities of typing methods for microorganisms. J Clin Microbiol 39: 4190-4192.

Huang CY, Lai JF, Huang IW, Chen PC, Wang HY, Shlau IR, Cheng YW, Hsleh LY, Chang SC, Lauderdale TSY 2014. Epidemiology and molecular characterization of macrolide-resistant Streptococcus pyogenes in Taiwan. J Clin Microbiol 52: 508-516.

Hunter PR, Gaston MA 1988. Numerical index of the discriminatory ability of typing systems: an application of Simpson's index of diversity. J Clin Microbiol 26: 2465-2466.

Mathur P, Bhardwaj N, Mathur K, Behera B, Gupta G, Kapil A, Singh S, Misra MC 2014. Clinical and molecular epidemiology of betahemolytic streptococcal infections in India. J Infect Dev Ctries 8: 297-303.

McNeil SA, Halperin SA, Langley JM, Smith B, Warren A, Sharratt GP, Baxendale DM, Reddish MA, Hu MC, Stroop SD, Linden J, Fries LF, Vink PE, Dale JB 2005. Safety and immunogenicity of 26-valent group A Streptococcus vaccine in healthy adult volunteers. Clin Infect Dis 41: 1114-1122.

Montes M, Tamayo E, Mojica C, García-Arenzana JM, Esnal O, Perez-Trallero E 2014. What causes decreased erythromycin resistance in Streptococcus pyogenes? Dynamics of four clones in a southern European region from 2005 to 2012. J Antimicrob Chemother 69: 1474-1482.

Montes M, Tamayo E, Orden B, Larruskain J, Perez-Trallero E 2010. Prevalence and clonal characterization of Streptococcus pyogenes clinical isolates with reduced fluoroquinolone susceptibility in Spain. Antimicrob Agents Chemother 54: 93-97.

Perez-Trallero E, Montes M, Orden B, Tamayo E, García-Arenzana JM, Marimón JM 2007. Phenotypic and genotypic characterization of Streptococcus pyogenes isolates displaying the $\mathrm{MLS}_{\mathrm{B}}$ phenotype of macrolide resistance in Spain, 1999 to 2005. Antimicrob Agents Chemother 51: 1228-1233.

Postol E, Alencar R, Higa FT, Barros SF, Demarchi LMF, Kalil J, Guilherme L 2013. StreptInCor: a candidate vaccine epitope against $S$. pyogenes infections induces protection in outbred mice. PLOS ONE 8: e60969.

Shea PR, Ewbank AL, Gonzalez-Lugo JH, Martagon-Rosado AJ, Martinez-Gutierrez JC, Rehman HA, Serrano-Gonzalez M, Fittipaldi N, Beres SB, Flores AR, Low DE, Willey BM, Musser JM 2011. Group A Streptococcus emm gene types in pharyngeal isolates, Ontario, Canada, 2002-2010. Emerg Infect Dis 17: 2010-2017.

Smeesters PR, Vergison A, Campos Jr D, Melderen LV 2009. Emerging fluoroquinolone-non-susceptible group A streptococci in two different paediatric populations. Int J Antimicrob Agents 34: 44-49.

Steer AC, Law I, Matatolu L, Beall BW, Carapetis JR 2009. Global emm type distribution of group A streptococci: systematic review and implications for vaccine development. Lancet Infect Dis 9: 611-616.

Sutcliffe J, Tait-Kamradt A, Wondrack L 1996. Streptococcus pneumoniae and Streptococcus pyogenes resistant to macrolides but sensitive to clindamycin: a common resistance pattern mediated by an efflux system. Antimicrob Agents Chemother 40: 1817-1824.

Syrogiannopoulos GA, Grivea IN, Al-Lahham A, Panagiotou M, Tsantouli AG, Reinert ANMRR, van der Linden M 2013. Sevenyear surveillance of emm types of pediatric group A streptococcal pharyngitis isolates in Western Greece. PLoS ONE 8: e71558.

Tartof SY, Reis JN, Andrade AN, Ramos RT, Reis MG, Riley LW 2010. Factors associated with Group A Streptococcus emm type diversification in a large urban setting in Brazil: a cross-sectional study. BMC Infect Dis 10: 327.

Teixeira LM, Barros RR, Castro ACD, Peralta JM, Carvalho MGS, Talkington DF, Vivoni AM, Facklam RR, Beall B 2001. Genetic and phenotypic features of Streptococcus pyogenes strains isolated in Brazil that harbor new emm sequences. J Clin Microbiol 39: 3290-3295.

Teixeira LM, Carvalho MG, Merquior VL, Steirgerwalt AG, Brenner DJ, Facklam RR 1997. Phenotypic and genotypic characterization of Vagococcus fluvialis, including strains isolated from human sources. J Clin Microbiol 35: 2778-2781.

Torres RSLA, Torres RPA, Smeesters PR, Palmeiro JK, Messias-Reason IJ, Dalla-Costa LM 2011. Group A Streptococcus antibiotic resistance in southern Brazil: a 17-year surveillance study. Microb Drug Resist 17: 313-319.

Whatmore AM, Kapur V, Sullivan DJ, Musser JM, Kehoe MA 1994. Non-congruent relationships between variation in emm gene sequences and the population genetic structure of group A streptococci. Mol Microbiol 14: 619-631. 